\title{
Exact radiation for dipoles on metallic spheroids at the interface between isorefractive half-spaces
}

\author{
Danilo Erricolo, Senior Member, IEEE, Piergiorgio L. E. Uslenghi, Fellow, IEEE
}

\begin{abstract}
Oblate and prolate metallic spheroids located at the interface between isorefractive half-spaces are considered. The electromagnetic fields produced by electric and magnetic dipoles located on the symmetry axis of the structure are determined exactly. Particular cases are discussed, and numerical results are presented for far fields and surface currents.
\end{abstract}

Index Terms-Electromagnetic radiation, complex media, spheroidal functions, isorefractive media

\section{INTRODUCTION}

$\mathbf{S}$ EVERAL boundary-value problems involving metallic structures, sometimes comprising sharp edges and cavities, and isorefractive media have recently been solved exactly in two and three dimensions [1],[2],[3],[4]. Numerical results based on these canonical solutions have been obtained and, for the structure considered in [1], have been compared to numerical solutions of integral-equation formulations of the same problem [5],[6],[7]. The excellent numerical agreements obtained establish the exact solutions as important benchmarks for the validation of frequency-domain computer codes.

In this paper, additional exact solutions are obtained for metallic oblate and prolate spheroids whose axis of symmetry is perpendicular to the planar interface that separates two isorefractive half-spaces and contains the center of symmetry of the spheroid. The primary source is an electric or magnetic dipole located on the axis of symmetry of the spheroid and axially oriented. The analysis is conducted in the frequency domain, and the time-dependent factor $\exp (-i \omega t)$ is omitted throughout. The exact electromagnetic field is found everywhere, and special attention is devoted to the far field and to the current density on the surface of the spheroid.

The geometry of the problem is illustrated in section II. The cases of the oblate and prolate spheroids are analyzed in Sections III and IV, respectively. Particular cases are briefly considered in Section V, and numerical results for surface currents and far fields are obtained, plotted and discussed in Section VI.

A detailed discussion of some practical applications of the results obtained in this paper is carried out in Section VII, where problems such as the buoy antenna and the groundstake antenna are briefly examined.

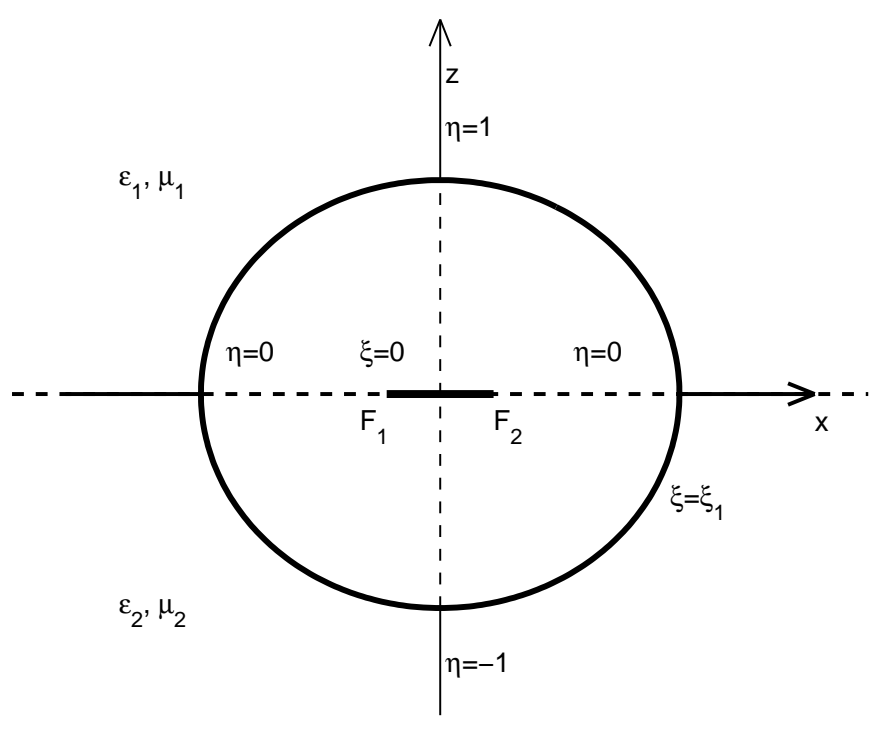

Fig. 1. Cross-section of a metallic oblate spheroid corresponding to the surface $\xi=\xi_{1}$ and located at the boundary between two isorefractive media. The segment joining the two foci $F_{1}$ and $F_{2}$ corresponds to $\xi=0$.

\section{GeOMETRY OF THE PROBLEM}

The geometry of the structures is shown in Fig. 1 for the oblate spheroid and in Fig. 2 for the prolate spheroid. Both spheroids are perfect electric conductors (PEC), have the $z$ axis of a rectangular coordinate system $(x, y, z)$ as axis of symmetry, and the center of the spheroid at the origin of coordinates. The $z=0$ plane is the interface that separates the half-space $z>0$, filled with a homogeneous and isotropic material of electric permittivity $\varepsilon_{1}$ and magnetic permeability $\mu_{1}$, from the half-space $z<0$, filled with a material of permittivity $\varepsilon_{2}$ and permeability $\mu_{2}$; we designate these two half-spaces as medium 1 and medium 2, respectively. The two media are isorefractive, i.e., they have the same propagation constant

$$
k=\omega \sqrt{\varepsilon_{h} \mu_{h}}, \quad(h=1,2)
$$

but, in general, different intrinsic impedances

$$
Z_{h}=Y_{h}^{-1}=\sqrt{\frac{\mu_{h}}{\varepsilon_{h}}} .
$$

The authors are with the Department of Electrical and Computer Engineering, University of Illinois at Chicago, Chicago, IL 60607 USA. Email: erricolo@ece.uic.edu, uslenghi@uic.edu. This work was supported by the U.S. Department of Defense under MURI grant F49620-01-1-0436 and by a grant of computer time from the DoD High Performance Computing Modernization Program at ASC and ERDC. 
In the case of the oblate spheroid of Fig. 1, we introduce the oblate spheroidal coordinates $(\eta, \xi, \varphi)$, with focal distance $d$, related to the rectangular coordinates $(x, y, z)$ by

$$
\left\{\begin{array}{l}
x=\frac{d}{2} \sqrt{\left(\xi^{2}+1\right)\left(1-\eta^{2}\right)} \cos \varphi \\
y=\frac{d}{2} \sqrt{\left(\xi^{2}+1\right)\left(1-\eta^{2}\right)} \sin \varphi \\
z=\frac{d}{2} \xi \eta
\end{array}\right.
$$

where $0 \leq \xi<\infty,-1 \leq \eta \leq 1,0 \leq \varphi \leq 2 \pi$. The inverse transformation from rectangular coordinates to oblate spheroidal coordinates is given in [2].

The disk $\xi=0$ has diameter $d$ and its edge is the focal circle of the oblate spheroidal coordinate system. The PEC oblate spheroid has surface $\xi=\xi_{1}$, the positive (negative) $z$ axis corresponds to $\eta=1(\eta=-1)$, and the portion of the $z=0$ plane that is exterior to the disk $\xi=0$ corresponds to $\eta=0$. The surfaces $\xi=$ constant are confocal oblate spheroids, the surfaces $|\eta|=$ constant are confocal one-sheet hyperboloids of revolution, and the surfaces $\varphi=$ constant are half-planes emanating from the $z$-axis.

In the case of the prolate spheroid of Fig. 2, we introduce the prolate spheroidal coordinates $(\eta, \xi, \varphi)$ related to the rectangular coordinates $(x, y, z)$ by

$$
\left\{\begin{array}{l}
x=\frac{d}{2} \sqrt{\left(\xi^{2}-1\right)\left(1-\eta^{2}\right)} \cos \varphi \\
y=\frac{d}{2} \sqrt{\left(\xi^{2}-1\right)\left(1-\eta^{2}\right)} \sin \varphi \\
z=\frac{d}{2} \xi \eta
\end{array}\right.
$$

where $1 \leq \xi<\infty,-1 \leq \eta \leq 1,0 \leq \varphi \leq 2 \pi$. The inverse transformation from rectangular coordinates to prolate

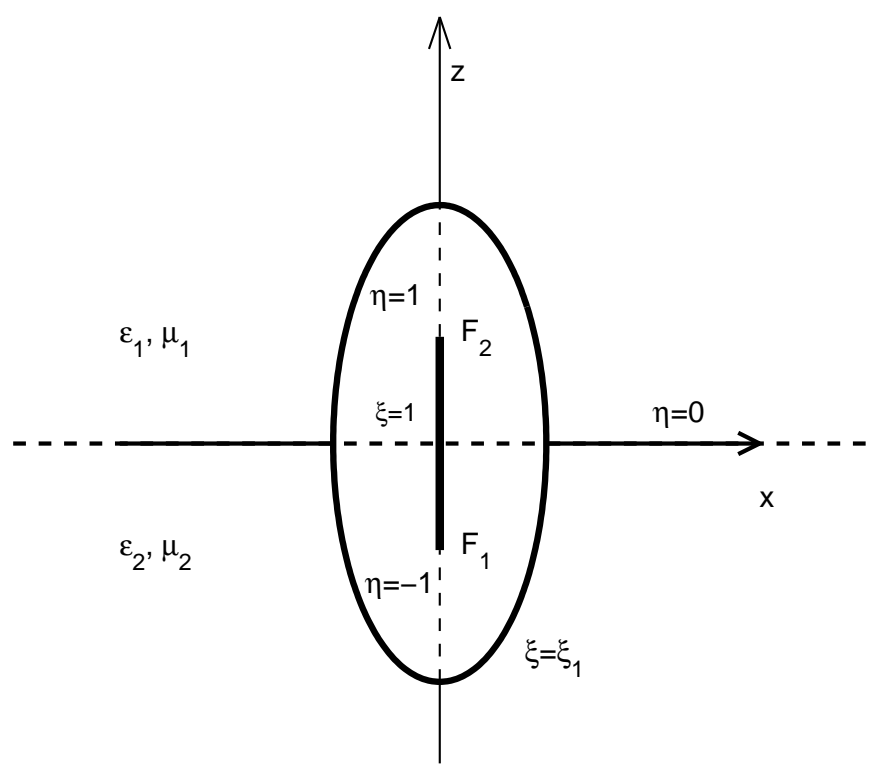

Fig. 2. Cross-section of a metallic prolate spheroid corresponding to the surface $\xi=\xi_{1}$ and located at the boundary between two isorefractive media. The segment joining the two foci $F_{1}$ and $F_{2}$ corresponds to the line $\xi=1$. spheroidal coordinates is:

$$
\left\{\begin{array}{l}
\xi=\sqrt{\frac{\left(4 r^{2}+d^{2}\right)+\sqrt{\left(4 r^{2}+d^{2}\right)^{2}-16 d^{2} z^{2}}}{2 d^{2}}} \\
\eta=\frac{2 z \sqrt{2}}{\sqrt{\left(4 r^{2}+d^{2}\right)+\sqrt{\left(4 r^{2}+d^{2}\right)^{2}-16 d^{2} z^{2}}}} \\
\varphi=\arctan \frac{y}{x} \quad\left(\text { where } r^{2}=x^{2}+y^{2}+z^{2}\right)
\end{array}\right.
$$

The segment $\xi=1$ has length $d$ and its end points $z= \pm d / 2$ are the foci of the prolate spheroidal coordinate system. The PEC prolate spheroid has surface $\xi=\xi_{1}$, the portion of the $z$ axis exterior to the two foci corresponds to $\eta=1$ for $z>d / 2$ and to $\eta=-1$ when $z<-d / 2$, and the interface $z=0$ to $\eta=0$. The surfaces $\xi=$ constant are confocal prolate spheroids, the surfaces $|\eta|=$ constant are confocal two-sheets hyperboloids of revolution, and the surfaces $\varphi=$ constant are half-planes emanating from the $z$-axis.

For both oblate and prolate spheroids, the primary source is a Hertzian electric or magnetic dipole located at $\left(\xi=\xi_{0} \geq\right.$ $\left.\xi_{1}, \eta=\eta_{0}=1\right)$ on the positive $z$-axis in medium 1 and oriented parallel to the $z$-axis. In all cases, the symbol $\xi_{>}\left(\xi_{<}\right)$ represents the larger (smaller) quantity between $\xi$ and $\xi_{0}$, and the parameter $c$ is given by:

$$
c=\frac{k d}{2}=\frac{\pi d}{\lambda}
$$

where $\lambda$ is the wavelength.

\section{THE OBLATE SPHEROID}

\section{A. Electric Dipole Source}

For an electric dipole at $\left(\xi_{0}, \eta_{0}=1\right)$ corresponding to an incident electric Hertz vector $\hat{z} \exp (i k R) /(k R)$, where $R$ is the distance from the dipole to the observation point, the incident magnetic field is oriented in the $\varphi$-direction and its $\varphi$-component is given by [8]:

$$
\begin{aligned}
H_{\varphi}^{i}= & \frac{2 k^{2} Y_{1}}{\sqrt{\xi_{0}^{2}+1}} \sum_{n=1}^{\infty} \frac{(-i)^{n}}{\widetilde{\rho}_{1, n} \widetilde{N}_{1, n}} \times \\
& R_{1, n}^{(1)}\left(-i c, i \xi_{<}\right) R_{1, n}^{(3)}\left(-i c, i \xi_{>}\right) S_{1, n}(-i c, \eta),
\end{aligned}
$$

where $R_{1, n}^{(1),(3)}$ are radial oblate spheroidal functions of the first and third kind, respectively, and $S_{1, n}$ are angular oblate spheroidal functions [9]. The quantities $\widetilde{\rho}_{1, n}$ and $\widetilde{N}_{1, n}$ are the normalization factors for oblate radial and angular spheroidal functions, respectively, of order 1 and degree $n$. Note that the summation in (7) starts from $n=1$, not $n=0$ as stated in [8].

The electric and magnetic fields $\left\{\underline{E}_{1}, \underline{H}_{1}\right\}$ in medium 1 and $\left\{\underline{E}_{2}, \underline{H}_{2}\right\}$ in medium 2 are:

$$
\left\{\begin{array}{l}
\underline{E}_{h}=E_{h \xi}(\xi, \eta) \hat{\xi}+E_{h \eta}(\xi, \eta) \hat{\eta} \\
\underline{H}_{h}=H_{h \varphi}(\xi, \eta) \hat{\varphi}, \quad(h=1,2)
\end{array},\right.
$$

where

$$
\begin{aligned}
& E_{h \xi}=-\frac{i Z_{h}}{c} \sqrt{\frac{1-\eta^{2}}{\xi^{2}+\eta^{2}}}\left(\frac{\partial}{\partial \eta}-\frac{\eta}{1-\eta^{2}}\right) H_{h \varphi}, \\
& E_{h \eta}=\frac{i Z_{h}}{c} \sqrt{\frac{\xi^{2}+1}{\xi^{2}+\eta^{2}}}\left(\frac{\partial}{\partial \xi}+\frac{\xi}{\xi^{2}+1}\right) H_{h \varphi} .
\end{aligned}
$$


The total magnetic field may be written as

$$
H_{1 \varphi}=H_{\varphi}^{i}+H_{\varphi}^{r}+H_{1 \varphi}^{s}, \quad H_{2 \varphi}=H_{\varphi}^{t}+H_{2 \varphi}^{s},
$$

where

$$
\begin{aligned}
& H_{\varphi}^{r}(\xi, \eta)=-R H_{\varphi}^{i}(\xi,-\eta), \\
& H_{\varphi}^{t}(\xi, \eta)=\zeta T H_{\varphi}^{i}(\xi, \eta)
\end{aligned}
$$

are the fields reflected and transmitted at the interface $z=0$ in the absence of the PEC spheroid. The electric-field reflection coefficient $R$ and transmission coefficient $T$ are given by

$$
R=\frac{1-\zeta}{1+\zeta}, \quad T=\frac{2}{1+\zeta}
$$

where

$$
\zeta=\frac{Z_{1}}{Z_{2}}
$$

Consequently,

$$
\begin{gathered}
H_{\varphi}^{i}+H_{\varphi}^{r}=\frac{2 k^{2} Y_{1}}{\sqrt{\xi_{0}^{2}+1}} \sum_{n=1}^{\infty} \frac{(-i)^{n}}{\widetilde{\rho}_{1, n} \widetilde{N}_{1, n}}\left[1+R(-1)^{n}\right] \times \\
R_{1, n}^{(1)}\left(-i c, i \xi_{<}\right) R_{1, n}^{(3)}\left(-i c, i \xi_{>}\right) S_{1, n}(-i c, \eta) .
\end{gathered}
$$

The scattered fields $H_{1 \varphi}^{s}$ in medium 1 and $H_{2 \varphi}^{s}$ in medium 2 are the perturbations introduced by the presence of the spheroid, and must satisfy the radiation condition:

$$
\begin{aligned}
H_{h \varphi}^{s}=\frac{2 k^{2} Y_{h}}{\sqrt{\xi_{0}^{2}+1}} \sum_{n=1}^{\infty} \frac{(-i)^{n} a_{h, n}}{\widetilde{\rho}_{1, n} \widetilde{N}_{1, n}} R_{1, n}^{(3)}\left(-i c, i \xi_{0}\right) \times \\
R_{1, n}^{(3)}(-i c, i \xi) S_{1, n}(-i c, \eta),(h=1,2) .
\end{aligned}
$$

The modal coefficients $a_{h, n}$ are found by imposing the boundary conditions on the surface of the spheroid and at the interface between the two media:

$$
\begin{aligned}
& a_{2, n}=\frac{T}{1+R(-1)^{n}} a_{1, n}= \\
& -\frac{T\left[\xi_{1} R_{1, n}^{(1)}\left(-i c, i \xi_{1}\right)+\left(\xi_{1}^{2}+1\right) R_{1, n}^{(1)^{\prime}}\left(-i c, i \xi_{1}\right)\right]}{A_{n}\left(c, \xi_{1}\right)}
\end{aligned}
$$

where

$$
A_{n}\left(c, \xi_{1}\right)=\xi_{1} R_{1, n}^{(3)}\left(-i c, i \xi_{1}\right)+\left(\xi_{1}^{2}+1\right) R_{1, n}^{(3)^{\prime}}\left(-i c, i \xi_{1}\right)
$$

and the prime means derivative with respect to $\xi$. In particular,

$$
a_{1,2 \ell}=a_{2,2 \ell}, \quad a_{1,2 \ell+1}=\zeta a_{2,2 \ell+1} .
$$

In considering the far field $(\xi \rightarrow \infty)$, it is convenient to introduce spherical polar coordinates $(r, \theta, \varphi)$ related to the rectangular coordinates $(x, y, z)$ in the usual manner. Then

$$
\left(H_{h \varphi}\right)_{\xi \rightarrow \infty} \sim \frac{e^{i k r}}{k r} F_{h \varphi}^{(e)}(\theta), \quad(h=1,2),
$$

where the far-field coefficients are given by:

$$
\begin{array}{r}
F_{1 \varphi}^{(e)}(\theta)=\frac{-2 i k^{2} Y_{1}}{\sqrt{\xi_{0}^{2}+1}} \sum_{n=1}^{\infty} \frac{(-1)^{n}}{\widetilde{\rho}_{1, n} \widetilde{N}_{1, n}}\left\{\left[1+R(-1)^{n}\right] \times\right. \\
\left.R_{1, n}^{(1)}\left(-i c, i \xi_{0}\right)+a_{1, n} R_{1, n}^{(3)}\left(-i c, i \xi_{0}\right)\right\} S_{1, n}(-i c, \cos \theta),(22)
\end{array}
$$

$$
\begin{aligned}
F_{2 \varphi}^{(e)}(\theta)= & \frac{-2 i k^{2} Y_{2}}{\sqrt{\xi_{0}^{2}+1}} \sum_{n=1}^{\infty} \frac{(-1)^{n}}{\widetilde{\rho}_{1, n} \widetilde{N}_{1, n}}\left\{T R_{1, n}^{(1)}\left(-i c, i \xi_{0}\right)+\right. \\
& \left.a_{2, n} R_{1, n}^{(3)}\left(-i c, i \xi_{0}\right)\right\} S_{1, n}(-i c, \cos \theta) ;
\end{aligned}
$$

note that (22) and (23) become identical when $Z_{1}=Z_{2}$. If the dipole is on the spheroid $\left(\xi_{0}=\xi_{1}\right)$, the far-field coefficients simplify to:

$$
\begin{gathered}
{\left[F_{1 \varphi}^{(e)}(\theta)\right]_{\xi_{0}=\xi_{1}}=\frac{2 k^{2} Y_{1}}{c \sqrt{\xi_{1}^{2}+1}} \sum_{n=1}^{\infty} \frac{\left[(-1)^{n}+R\right] S_{1, n}(-i c, \cos \theta)}{\widetilde{\rho}_{1, n} \widetilde{N}_{1, n} A_{n}\left(c, \xi_{1}\right)},} \\
{\left[F_{2 \varphi}^{(e)}(\theta)\right]_{\xi_{0}=\xi_{1}}=\frac{2 k^{2} Y_{2} T}{c \sqrt{\xi_{1}^{2}+1}} \sum_{n=1}^{\infty} \frac{(-1)^{n} S_{1, n}(-i c, \cos \theta)}{\widetilde{\rho}_{1, n} \widetilde{N}_{1, n} A_{n}\left(c, \xi_{1}\right)} .}
\end{gathered}
$$

The surface current densities on the PEC spheroid are easily found from the total magnetic field tangent to the surface:

$$
\begin{aligned}
\left(H_{1 \varphi}\right)_{\xi=\xi_{1}}= & \frac{2 i k^{2} Y_{1}}{c \sqrt{\xi_{0}^{2}+1}} \sum_{n=1}^{\infty} \frac{(-i)^{n}\left[1+R(-1)^{n}\right]}{\widetilde{\rho}_{1, n} \widetilde{N}_{1, n} A_{n}\left(c, \xi_{1}\right)} \times \\
& R_{1, n}^{(3)}\left(-i c, i \xi_{0}\right) S_{1, n}(-i c, \eta), \\
\left(H_{2 \varphi}\right)_{\xi=\xi_{1}}= & \frac{2 i k^{2} Y_{2} T}{c \sqrt{\xi_{0}^{2}+1}} \sum_{n=1}^{\infty} \frac{(-i)^{n}}{\widetilde{\rho}_{1, n} \widetilde{N}_{1, n} A_{n}\left(c, \xi_{1}\right)} \times \\
& R_{1, n}^{(3)}\left(-i c, i \xi_{0}\right) S_{1, n}(-i c, \eta) .
\end{aligned}
$$

\section{B. Magnetic Dipole Source}

For a magnetic dipole at $\left(\xi_{0}, \eta_{0}=1\right)$ corresponding to an incident magnetic Hertz vector $\hat{z} \exp (i k R) /(k R)$, the incident electric field is oriented in the $\varphi$-direction and its $\varphi$-component is given by [8]:

$$
\begin{aligned}
E_{\varphi}^{i}= & \frac{-2 k^{2} Z_{1}}{\sqrt{\xi_{0}^{2}+1}} \sum_{n=1}^{\infty} \frac{(-i)^{n}}{\widetilde{\rho}_{1, n} \widetilde{N}_{1, n}} \times \\
& R_{1, n}^{(1)}\left(-i c, i \xi_{<}\right) R_{1, n}^{(3)}\left(-i c, i \xi_{>}\right) S_{1, n}(-i c, \eta) .
\end{aligned}
$$

The electric and magnetic fields $\left\{\underline{E}_{1}, \underline{H}_{1}\right\}$ in medium 1 and $\left\{\underline{E}_{2}, \underline{H}_{2}\right\}$ in medium 2 are:

$$
\left\{\begin{array}{l}
\underline{E}_{h}=E_{h \varphi} \hat{\varphi} \\
\underline{H}_{h}=H_{h \xi}(\xi, \eta) \hat{\xi}+H_{h \eta}(\xi, \eta) \hat{\eta}, \quad(h=1,2)
\end{array}\right.
$$

where

$$
\begin{gathered}
H_{h \xi}=\frac{i Y_{h}}{c} \sqrt{\frac{1-\eta^{2}}{\xi^{2}+\eta^{2}}}\left(\frac{\partial}{\partial \eta}-\frac{\eta}{1-\eta^{2}}\right) E_{h \varphi}, \\
H_{h \eta}=-\frac{i Y_{h}}{c} \sqrt{\frac{\xi^{2}+1}{\xi^{2}+\eta^{2}}}\left(\frac{\partial}{\partial \xi}+\frac{\xi}{\xi^{2}+1}\right) E_{h \varphi} .
\end{gathered}
$$

The total electric field may be written as

$$
E_{1 \varphi}=E_{\varphi}^{i}+E_{\varphi}^{r}+E_{1 \varphi}^{s}, \quad E_{2 \varphi}=E_{\varphi}^{t}+E_{2 \varphi}^{s},
$$

where

$$
\begin{aligned}
& E_{\varphi}^{r}(\xi, \eta)=R E_{\varphi}^{i}(\xi,-\eta), \\
& E_{\varphi}^{t}(\xi, \eta)=T E_{\varphi}^{i}(\xi, \eta)
\end{aligned}
$$


are the fields reflected and transmitted at $z=0$ in the absence of the spheroid. Consequently,

$$
\begin{aligned}
E_{\varphi}^{i}+E_{\varphi}^{r}= & \frac{-2 k^{2} Z_{1}}{\sqrt{\xi_{0}^{2}+1}} \sum_{n=1}^{\infty} \frac{(-i)^{n}}{\widetilde{\rho}_{1, n} \widetilde{N}_{1, n}}\left[1-R(-1)^{n}\right] \times \\
& R_{1, n}^{(1)}\left(-i c, i \xi_{<}\right) R_{1, n}^{(3)}\left(-i c, i \xi_{>}\right) S_{1, n}(-i c, \eta) .
\end{aligned}
$$

The scattered fields $E_{1 \varphi}^{s}$ in medium 1 and $E_{2 \varphi}^{s}$ in medium 2 are the perturbations introduced by the presence of the spheroid:

$$
\begin{aligned}
E_{h \varphi}^{s}= & \frac{-2 k^{2} Z_{h}}{\sqrt{\xi_{0}^{2}+1}} \sum_{n=1}^{\infty} \frac{(-i)^{n} b_{h, n}}{\widetilde{\rho}_{1, n} \widetilde{N}_{1, n}} R_{1, n}^{(3)}\left(-i c, i \xi_{0}\right) \times \\
& R_{1, n}^{(3)}(-i c, i \xi) S_{1, n}(-i c, \eta), \quad(h=1,2)
\end{aligned}
$$

where the modal coefficients $b_{h, n}$ are found to be given by:

$$
b_{2, n}=\frac{\zeta T}{1-R(-1)^{n}} b_{1, n}=-\zeta T \frac{R_{1, n}^{(1)}\left(-i c, i \xi_{1}\right)}{R_{1, n}^{(3)}\left(-i c, i \xi_{1}\right)} ;
$$

in particular,

$$
b_{2,2 \ell}=b_{1,2 \ell}, \quad b_{2,2 \ell+1}=\zeta b_{1,2 \ell+1} .
$$

In the far field,

$$
\left(E_{h \varphi}\right)_{\xi \rightarrow \infty} \sim \frac{e^{i k r}}{k r} F_{h \varphi}^{(m)}(\theta), \quad(h=1,2),
$$

where

$$
\begin{gathered}
F_{1 \varphi}^{(m)}(\theta)=\frac{2 i k^{2} Z_{1}}{\sqrt{\xi_{0}^{2}+1}} \sum_{n=1}^{\infty} \frac{(-1)^{n}}{\widetilde{\rho}_{1, n} \widetilde{N}_{1, n}}\left\{\left[1-R(-1)^{n}\right] \times\right. \\
\left.R_{1, n}^{(1)}\left(-i c, i \xi_{0}\right)+b_{1, n} R_{1, n}^{(3)}\left(-i c, i \xi_{0}\right)\right\} S_{1, n}(-i c, \cos \theta), \quad(40) \\
F_{2 \varphi}^{(m)}(\theta)=\frac{2 i k^{2} Z_{2}}{\sqrt{\xi_{0}^{2}+1}} \sum_{n=1}^{\infty} \frac{(-1)^{n}}{\widetilde{\rho}_{1, n} \widetilde{N}_{1, n}}\left\{\zeta T R_{1, n}^{(1)}\left(-i c, i \xi_{0}\right)+\right. \\
\left.b_{2, n} R_{1, n}^{(3)}\left(-i c, i \xi_{0}\right)\right\} S_{1, n}(-i c, \cos \theta) ;
\end{gathered}
$$

note that (40) and (41) become identical when $Z_{1}=Z_{2}$.

The surface current densities on the spheroid are found from the total magnetic field tangent to the surface:

$$
\begin{gathered}
\left(H_{1 \eta}\right)_{\xi=\xi_{1}}=\frac{2 k^{2}}{c^{2} \sqrt{\left(\xi_{0}^{2}+1\right)\left(\xi_{1}^{2}+1\right)\left(\xi_{1}^{2}+\eta^{2}\right)}} \times \\
\sum_{n=1}^{\infty} \frac{(-i)^{n}\left[1-R(-1)^{n}\right]}{\widetilde{\rho}_{1, n}} \frac{R_{1, n}^{(3)}\left(-i c, i \xi_{0}\right)}{\widetilde{N}_{1, n}} S_{1, n}(-i c, \eta), \\
\left(H_{2 \eta}\right)_{\xi=\xi_{1}}=\frac{2 k^{2} \zeta T}{c^{2} \sqrt{\left(\xi_{0}^{2}+1\right)\left(\xi_{1}^{2}+1\right)\left(\xi_{1}^{2}+\eta^{2}\right)}} \times \\
\sum_{n=1}^{\infty} \frac{(-i)^{n}}{\widetilde{\rho}_{1, n} \widetilde{N}_{1, n}} \frac{R_{1, n}^{(3)}\left(-i c, i \xi_{0}\right)}{R_{1, n}^{(3)}\left(-i c, i \xi_{1}\right)} S_{1, n}(-i c, \eta),
\end{gathered}
$$

It should be noted that in deriving the surface fields (42-43), as well as (26-27) for the case of the electric dipole source, it was assumed that $\xi<\xi_{0}$. If we had assumed $\xi>\xi_{0}$ and then let $\xi_{0} \rightarrow \xi_{1}$, i.e. sent the magnetic dipole to the surface of the spheroid, then all fields everywhere would be zero.

\section{The Prolate SPHERoID}

Since derivations are similar to those for the oblate spheroid, details are omitted and only the results are given.

\section{A. Electric Dipole Source}

For the same electric dipole considered for the oblate spheroid, the $\varphi$ component of the incident magnetic field may be expanded in a series of prolate spheroidal wave functions, with notation as in [8], [9]:

$$
\begin{aligned}
H_{\varphi}^{i}= & \frac{2 k^{2} Y_{1}}{\sqrt{\xi_{0}^{2}-1}} \sum_{n=1}^{\infty} \frac{(-i)^{n-1}}{\rho_{1, n} N_{1, n}} \times \\
& R_{1, n}^{(1)}\left(c, \xi_{<}\right) R_{1, n}^{(3)}\left(c, \xi_{>}\right) S_{1, n}(c, \eta),
\end{aligned}
$$

where $\rho_{1, n}$ and $N_{1, n}$ are the normalization factors for prolate radial and angular spheroidal functions, respectively, of order 1 and degree $n$. Equations (8) and (11-15) remain valid, while (9-10) are replaced by

$$
\begin{gathered}
E_{h \xi}=-\frac{i Z_{h}}{c} \sqrt{\frac{1-\eta^{2}}{\xi^{2}-\eta^{2}}}\left(\frac{\partial}{\partial \eta}-\frac{\eta}{1-\eta^{2}}\right) H_{h \varphi}, \\
E_{h \eta}=\frac{i Z_{h}}{c} \sqrt{\frac{\xi^{2}-1}{\xi^{2}-\eta^{2}}}\left(\frac{\partial}{\partial \xi}+\frac{\xi}{\xi^{2}+1}\right) H_{h \varphi} .
\end{gathered}
$$

It is found that

$$
\begin{aligned}
H_{\varphi}^{i}+H_{\varphi}^{r}= & \left.\frac{2 k^{2} Y_{1}}{\sqrt{\xi_{0}^{2}-1}} \sum_{n=1}^{\infty} \frac{(-i)^{n-1}}{\rho_{1, n} N_{1, n}}\left[1+R(-1)^{n}\right)\right] \times \\
& R_{1, n}^{(1)}\left(c, \xi_{<}\right) R_{1, n}^{(3)}\left(c, \xi_{>}\right) S_{1, n}(c, \eta), \\
H_{h \varphi}^{s}= & \frac{2 k^{2} Y_{h}}{\sqrt{\xi_{0}^{2}-1}} \sum_{n=1}^{\infty} \frac{(-i)^{n-1}}{\rho_{1, n} N_{1, n}} c_{h, n} \times \\
& R_{1, n}^{(3)}\left(c, \xi_{0}\right) R_{1, n}^{(3)}(c, \xi) S_{1, n}(c, \eta),
\end{aligned}
$$

where

$$
\begin{aligned}
c_{2, n} & =\frac{T}{1+R(-1)^{n}} c_{1, n}= \\
- & \frac{T\left[\xi_{1} R_{1, n}^{(1)}\left(c, \xi_{1}\right)+\left(\xi_{1}^{2}-1\right) R_{1, n}^{(1)^{\prime}}\left(c, \xi_{1}\right)\right]}{C_{n}\left(c, \xi_{1}\right)},
\end{aligned}
$$

with

$$
C_{n}\left(c, \xi_{1}\right)=\xi_{1} R_{1, n}^{(3)}\left(c, \xi_{1}\right)+\left(\xi_{1}^{2}-1\right) R_{1, n}^{(3)^{\prime}}\left(c, \xi_{1}\right) .
$$

In the far field,

$$
\left(H_{h \varphi}\right)_{\xi \rightarrow \infty} \sim \frac{e^{i k r}}{k r} G_{h \varphi}^{(e)}(\theta), \quad(h=1,2),
$$

where

$$
\begin{aligned}
& G_{1 \varphi}^{(e)}(\theta)= \frac{2 k^{2} Y_{1}}{\sqrt{\xi_{0}^{2}-1}} \sum_{n=1}^{\infty} \frac{(-1)^{n}}{\rho_{1, n} N_{1, n}}\left\{\left[1+R(-1)^{n}\right] \times\right. \\
&\left.R_{1, n}^{(1)}\left(c, \xi_{0}\right)+c_{1, n} R_{1, n}^{(3)}\left(c, \xi_{0}\right)\right\} S_{1, n}(c, \cos \theta), \\
& G_{2 \varphi}^{(e)}(\theta)=\frac{2 k^{2} Y_{2}}{\sqrt{\xi_{0}^{2}-1}} \sum_{n=1}^{\infty} \frac{(-1)^{n}}{\rho_{1, n} N_{1, n}}\left\{T R_{1, n}^{(1)}\left(c, \xi_{0}\right)+\right. \\
&\left.c_{2, n} R_{1, n}^{(3)}\left(c, \xi_{0}\right)\right\} S_{1, n}(c, \cos \theta) ;
\end{aligned}
$$


note that (52) and (53) become identical when $Z_{1}=Z_{2}$. If the dipole is on the spheroid,

$$
\begin{gathered}
{\left[G_{1 \varphi}^{(e)}(\theta)\right]_{\xi_{0}=\xi_{1}}=\frac{2 i k^{2} Y_{1}}{c \sqrt{\xi_{1}^{2}-1}} \sum_{n=1}^{\infty} \frac{\left[(-1)^{n}+R\right] S_{1, n}(c, \cos \theta)}{\rho_{1, n} N_{1, n} C_{n}\left(c, \xi_{1}\right)}} \\
{\left[G_{2 \varphi}^{(e)}(\theta)\right]_{\xi_{0}=\xi_{1}}=\frac{2 i k^{2} Y_{2}}{c \sqrt{\xi_{1}^{2}-1}} T \sum_{n=1}^{\infty} \frac{(-1)^{n} S_{1, n}(c, \cos \theta)}{\rho_{1, n} N_{1, n} C_{n}\left(c, \xi_{1}\right)}}
\end{gathered}
$$

The surface current densities on the spheroid are found from the total magnetic field tangent to the surface:

$$
\begin{aligned}
& \left(H_{1 \varphi}\right)_{\xi=\xi_{1}}=-\frac{2 k^{2} Y_{1}}{c \sqrt{\xi_{0}^{2}-1}} \sum_{n=1}^{\infty} \frac{(-i)^{n}\left[1+R(-1)^{n}\right]}{\rho_{1, n} N_{1, n} C_{n}\left(c, \xi_{1}\right)} \times \\
& R_{1, n}^{(3)}\left(c, \xi_{0}\right) S_{1, n}(c, \eta) \\
& \left(H_{2 \varphi}\right)_{\xi=\xi_{1}}=-\frac{2 k^{2} Y_{2}}{c \sqrt{\xi_{0}^{2}-1}} T \sum_{n=1}^{\infty} \frac{(-i)^{n}}{\rho_{1, n} N_{1, n} C_{n}\left(c, \xi_{1}\right)} \times \\
& R_{1, n}^{(3)}\left(c, \xi_{0}\right) S_{1, n}(c, \eta)
\end{aligned}
$$

\section{B. Magnetic Dipole Source}

For the same magnetic dipole considered for the oblate spheroid, the $\varphi$ component of the incident electric field may be expanded in a series of prolate spheroidal wave functions, with notation as in [8][9]:

$$
\begin{aligned}
E_{\varphi}^{i}= & \frac{-2 k^{2} Z_{1}}{\sqrt{\xi_{0}^{2}-1}} \sum_{n=1}^{\infty} \frac{(-i)^{n-1}}{\rho_{1, n} N_{1, n}} \times \\
& R_{1, n}^{(1)}\left(c, \xi_{<}\right) R_{1, n}^{(3)}\left(c, \xi_{>}\right) S_{1, n}(c, \eta) .
\end{aligned}
$$

Equations (29) and (32-34) still hold, while (30-31) are replaced by

$$
\begin{gathered}
H_{h \xi}=\frac{i Y_{h}}{c} \sqrt{\frac{1-\eta^{2}}{\xi^{2}-\eta^{2}}}\left(\frac{\partial}{\partial \eta}-\frac{\eta}{1-\eta^{2}}\right) E_{h \varphi}, \\
H_{h \eta}=-\frac{i Y_{h}}{c} \sqrt{\frac{\xi^{2}-1}{\xi^{2}-\eta^{2}}}\left(\frac{\partial}{\partial \xi}+\frac{\xi}{\xi^{2}-1}\right) E_{h \varphi} .
\end{gathered}
$$

It is found that

$$
\begin{aligned}
E_{\varphi}^{i}+E_{\varphi}^{r}= & \frac{-2 k^{2} Z_{1}}{\sqrt{\xi_{0}^{2}-1}} \sum_{n=1}^{\infty} \frac{(-i)^{n-1}}{\rho_{1, n} N_{1, n}}\left[1-R(-1)^{n}\right] \times \\
& R_{1, n}^{(1)}\left(c, \xi_{<}\right) R_{1, n}^{(3)}\left(c, \xi_{>}\right) S_{1, n}(c, \eta), \\
E_{h \varphi}^{s}= & \frac{-2 k^{2} Z_{h}}{\sqrt{\xi_{0}^{2}-1}} \sum_{n=1}^{\infty} \frac{(-i)^{n-1}}{\rho_{1, n} N_{1, n}} d_{h, n} \times \\
& R_{1, n}^{(3)}\left(c, \xi_{0}\right) R_{1, n}^{(3)}(c, \xi) S_{1, n}(c, \eta)
\end{aligned}
$$

where

$$
d_{2, n}=\frac{\zeta T}{1-R(-1)^{n}} d_{1, n}=-\zeta T \frac{R_{1, n}^{(1)}\left(c, \xi_{1}\right)}{R_{1, n}^{(3)}\left(c, \xi_{1}\right)},
$$

so that, in particular,

$$
d_{2,2 \ell}=d_{1,2 \ell}, \quad d_{2,2 \ell+1}=\zeta d_{1,2 \ell+1} .
$$

In the far field,

$$
\left(E_{h \varphi}\right)_{\xi \rightarrow \infty} \sim \frac{e^{i k r}}{k r} G_{h \varphi}^{(m)}(\theta), \quad(h=1,2),
$$

where

$$
\begin{aligned}
& G_{1 \varphi}^{(m)}(\theta)= \frac{-2 k^{2} Z_{1}}{\sqrt{\xi_{0}^{2}-1}} \sum_{n=1}^{\infty} \frac{(-1)^{n}}{\rho_{1, n} N_{1, n}}\left\{\left[1-R(-1)^{n}\right] \times\right. \\
&\left.R_{1, n}^{(1)}\left(c, \xi_{0}\right)+d_{1, n} R_{1, n}^{(3)}\left(c, \xi_{0}\right)\right\} S_{1, n}(c, \cos \theta), \\
& G_{2 \varphi}^{(m)}(\theta)=\frac{-2 k^{2} Z_{2}}{\sqrt{\xi_{0}^{2}-1}} \sum_{n=1}^{\infty} \frac{(-1)^{n}}{\rho_{1, n} N_{1, n}}\left\{\zeta T R_{1, n}^{(1)}\left(c, \xi_{0}\right)+\right. \\
&\left.d_{2, n} R_{1, n}^{(3)}\left(c, \xi_{0}\right)\right\} S_{1, n}(c, \cos \theta) ;
\end{aligned}
$$

note that (66) and (67) become identical when $Z_{1}=Z_{2}$.

The surface current densities on the spheroid are found from the total magnetic field tangent to the surface:

$$
\begin{gathered}
\left(H_{1 \eta}\right)_{\xi=\xi_{1}}=\frac{2 k^{2}}{c^{2} \sqrt{\left(\xi_{0}^{2}-1\right)\left(\xi_{1}^{2}-1\right)\left(\xi_{1}^{2}-\eta^{2}\right)}} \times \\
\sum_{n=1}^{\infty} \frac{(-i)^{n-1}\left[1-R(-1)^{n}\right]}{\rho_{1, n} N_{1, n}} \frac{R_{1, n}^{(3)}\left(c, \xi_{0}\right)}{R_{1, n}^{(3)}\left(c, \xi_{1}\right)} S_{1, n}(c, \eta), \\
\left(H_{2 \eta}\right)_{\xi=\xi_{1}}=\frac{2 k^{2} \zeta T}{c^{2} \sqrt{\left(\xi_{0}^{2}-1\right)\left(\xi_{1}^{2}-1\right)\left(\xi_{1}^{2}-\eta^{2}\right)}} \times \\
\sum_{n=1}^{\infty} \frac{(-i)^{n-1}}{\rho_{1, n} N_{1, n}} \frac{R_{1, n}^{(3)}\left(c, \xi_{0}\right)}{R_{1, n}^{(3)}\left(c, \xi_{1}\right)} S_{1, n}(c, \eta) .
\end{gathered}
$$

\section{Particular Cases}

The case of the sphere may be obtained as a limiting case from either the oblate or the prolate spheroid cases, when $d \rightarrow$ 0 (hence $c \rightarrow 0$ ). The specific results are not reported here. The case of the wire cannot be easily obtained from the results for the prolate spheroid as $\xi_{1} \rightarrow 1$ because $\xi=1$ is a singular point for the coefficients of the differential equation satisfied by the radial prolate spheroidal functions. On the other hand, the case of the circular disk corresponds to the limit $\xi_{1}=0$ for the oblate spheroid, and this is not a singular point for the coefficients of the differential equation satisfied by the radial oblate spheroidal functions. Consequently, the circular disk is easily treated as a particular case for the oblate spheroid, by letting $\xi_{1}=0$ in the formulas of Section III. In particular, the coefficients (18) simplify to:

$$
\begin{aligned}
\left(a_{h, 2 \ell+1}\right)_{\xi_{1}=0} & =0 \\
\left(a_{h, 2 \ell}\right)_{\xi_{1}=0} & =-T \frac{R_{1,2 \ell}^{(1)^{\prime}}(-i c, i 0)}{R_{1,2 \ell}^{(3)^{\prime}}(-i c, i 0)},
\end{aligned}
$$

whereas the coefficients (37) become:

$$
\begin{aligned}
\left(b_{h, 2 \ell}\right)_{\xi_{1}=0} & =0 \\
\left(b_{2,2 \ell+1}\right)_{\xi_{1}=0} & =\zeta\left(b_{1,2 \ell+1}\right)_{\xi_{1}=0}=-\zeta T \frac{R_{1,2 \ell+1}^{(1)}(-i c, i 0)}{R_{1,2 \ell+1}^{(3)}(-i c, i 0)} .
\end{aligned}
$$




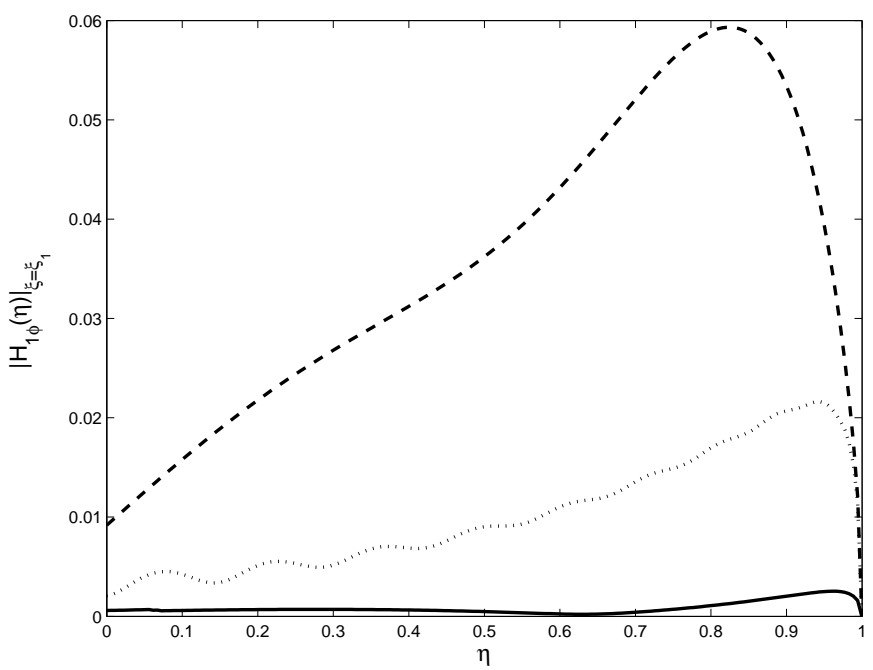

Fig. 3. Surface current density for an oblate spheroid with $\xi_{1}=2$ due to an electric dipole located at $\left(\xi_{0}=3, \eta_{0}=1\right)$ when $Z_{1}=120 \pi \Omega$ and $Z_{2}=240 \pi \Omega$. Results show $c=1$ (solid line), $c=\pi$ (dashed line), and $c=10$ (dotted line).

\section{NumERICAL RESUlTS}

The numerical results obtained in this section and exhibited in Figs. 3-6 are provided as test cases for researchers who may want to check the accuracy of their computer codes without becoming embroiled in the numerical evaluation of series of spheroidal functions.

Computations of the spheroidal functions were performed using some of the Fortran subroutines reported in [10]. The numerical examples discussed in the following all refer to a dipole source located on the positive $z$-axis at the spheroidal coordinate $\xi_{0}=3$. For both oblate and prolate cases, the metallic spheroid corresponds to the coordinate surface $\xi_{1}=2$. The values of the intrinsic impedances of the isorefractive media are indicated in the captions to the figures. For each case examined, three values of the parameter $c$, defined in (6), are investigated: $c=1, \pi, 10$.

Fig. 3 shows the current induced on the surface of an oblate spheroid due to an electric dipole, computed using (26).

Fig. 4 shows the far-field coefficient due to an electric dipole in the presence of an oblate spheroid, computed using (22).

Fig. 5 shows the far-field coefficient due to an electric dipole in the presence of a prolate spheroid, computed using (52). Even though Figs. 4 and 5 appear similar, one should observe that in Fig. $4 Z_{2}=240 \pi \Omega$, while in Fig. $5 Z_{2}=360 \pi \Omega$.

Finally, Fig. 6 shows the far field coefficient due to a magnetic dipole in the presence of a prolate spheroid computed using (66). In Figs. 4, 5, 6 the independent variable is the spherical coordinate elevation angle $\theta$, which is related to the spheroidal coordinate $\eta$ by $\eta=\cos \theta$. Hence, when $\theta \rightarrow 0$, the far-field coefficient vanishes as one would expect from the behavior of the field of a dipole along its axis.

These results were computed by summing the series using the acceleration technique described in [11], which is based on Shanks' transform. The number of series terms that were actually summed was determined by the convergence criterion for each specific summation. The convergence criterion that was adopted essentially required that the relative variation of three consecutive approximations of the sum were all below a threshold that was set to $10^{-6}$. By so doing, at most the first 58 terms of any series were computed. Additionally, the computations were repeated by executing the Fortran subroutines using quadruple precision variables and the algorithm was forced to always compute at least 100 terms and with an even lower threshold, but no appreciable variations were observed in the numerical results, which confirmed the accuracy of the original computations. The computation time, for double precision variables, is in the order of a few minutes using an ordinary Windows based PC, with a clock frequency of $1.5 \mathrm{GHz}$ and $768 \mathrm{MB}$ of RAM memory.

\section{DISCUSSION AND CONCLUSION}

Analytical results for a new canonical problem involving a metallic spheroid, two isorefractive media and a dipole source located along the axis of symmetry of revolution were obtained. This new analytical solution enriches the list of electromagnetic problems for which exact solutions are known. Numerical results for induced currents and far-field coefficients were provided and discussed. This solution is important, for instance, to conduct comparisons with other methods, similar to what is discussed in [5], [6], [7].

There are several practical antenna configurations whose analysis can benefit from the results obtained in this paper. The buoy antenna, consisting of an antenna axially mounted on a body of revolution floating in water, is not amenable to exact analytical solutions and is often analyzed numerically. Even though lake and sea water are not isorefractive to air and, furthermore, non-negligible losses occur especially in sea water, those researchers who desire to check their computer code against an exact result could adjust their parameters to fit solutions presented in this paper and verify their computed data at least in this particular case. The ground-stake antenna

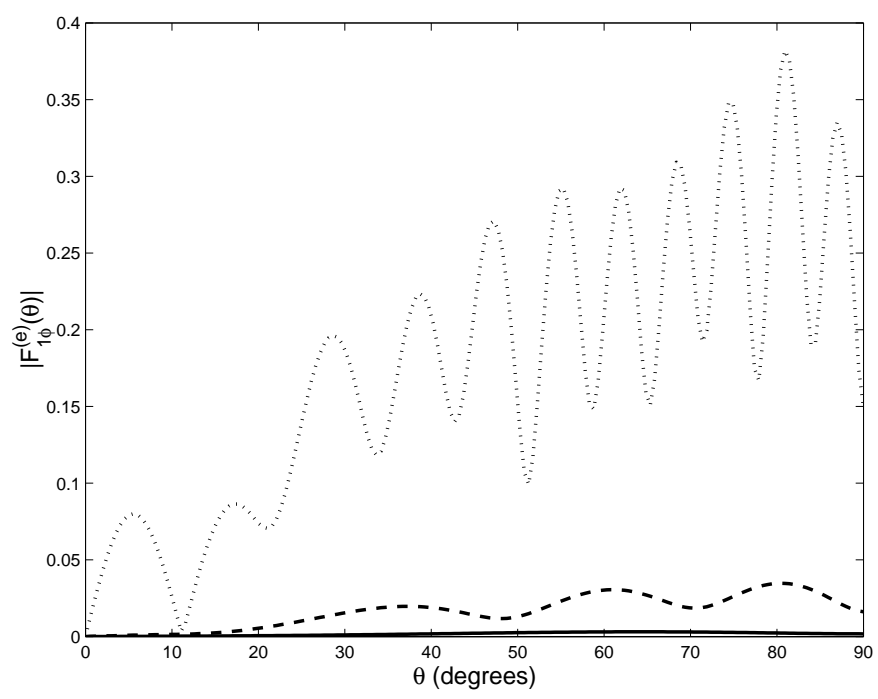

Fig. 4. Far-field coefficient for an oblate spheroid with $\xi_{1}=2$ due to an electric dipole located at $\left(\xi_{0}=3, \eta_{0}=1\right)$ when $Z_{1}=120 \pi \Omega$ and $Z_{2}=240 \pi \Omega$. Results show $c=1$ (solid line), $c=\pi$ (dashed line), and $c=10$ (dotted line). 


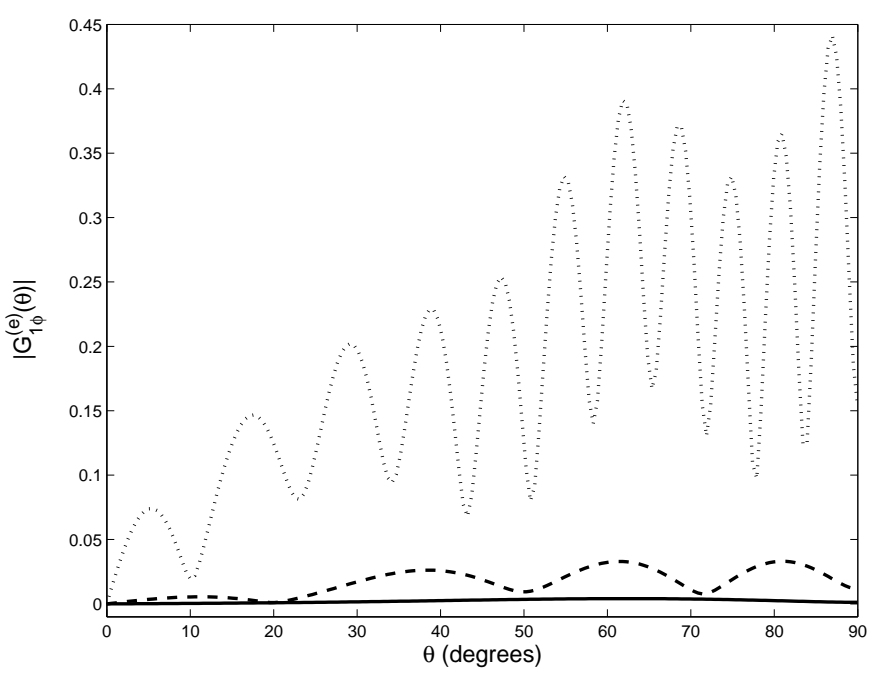

Fig. 5. Far-field coefficient for a prolate spheroid with $\xi_{1}=2$ due to an electric dipole located at $\left(\xi_{0}=3, \eta_{0}=1\right)$ when $Z_{1}=120 \pi \Omega$ and $Z_{2}=360 \pi \Omega$. Results show $c=1$ (solid line), $c=\pi$ (dashed line), and $c=10$ (dotted line).

is a thin wire antenna oriented perpendicular to the interface between two different media and located partially in either medium. The analysis of this antenna is a practically important but difficult problem that has attracted a lot of attention and has been solved either numerically or asymptotically [1217]. In asymptotic studies, the evaluation of Sommerfeld-type integrals is especially delicate [18]. A related problem is that of a linear antenna on the axis of a circular aperture in a planar screen separating two different media [19]. Computer codes providing solutions for this class of problems may be checked against the exact results of Section IV of this paper by simulating the wire antenna with a thin prolate spheroid and taking the two media to be isorefractive. Again, this is

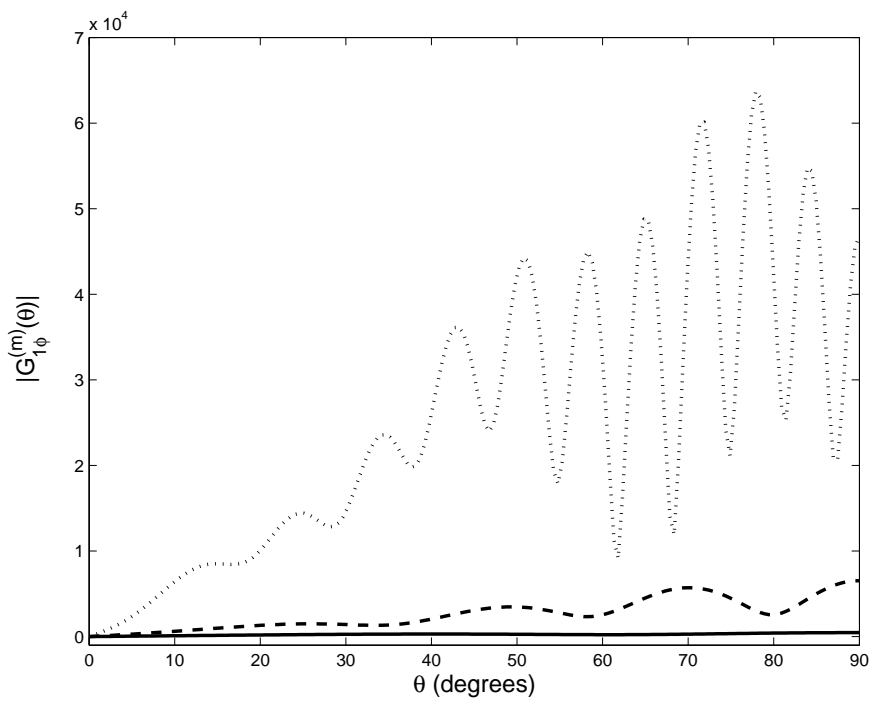

Fig. 6. Far-field coefficient for a prolate spheroid with $\xi_{1}=2$ due to a magnetic dipole located at $\left(\xi_{0}=3, \eta_{0}=1\right)$ when $Z_{1}=120 \pi \Omega$ and $Z_{2}=360 \pi \Omega$. Results show $c=1$ (solid line), $c=\pi$ (dashed line), and $c=10$ (dotted line). only a partial check for a very specific set of parameters, but is the only verification available that is based on an exact solution. Another important problem is that of a vertical wire antenna mounted on a PEC platform located on a lossy ground. Asymptotic solutions have been developed, but their accuracy may be questionable if the edges of the platform are not in the far field of the antenna, so a numerical approach may be more accurate. Any asymptotic or numerical solution developed for this system may be checked by comparison with our exact result of Section V, when the PEC platform is a circular disk and the ground is taken as isorefractive to air.

\section{ACKNOWLEDGMENTS}

The authors are grateful to Prof. C. M. Butler for bringing several papers to their attention, and to both him and Dr. W. A. Johnson for helpful discussions.

\section{REFERENCES}

[1] P.L.E. Uslenghi, "Exact penetration, radiation and scattering for a slotted semielliptical channel filled with isorefractive material," IEEE Trans. Antennas Propagat., vol. 52, no. 6, pp. 1473-1480, June 2004.

[2] C. Berardi, D. Erricolo, and P.L.E. Uslenghi, "Exact dipole radiation for an oblate spheroidal cavity filled with isorefractive material and aperturecoupled to a half space," IEEE Trans. Antennas Propagat., vol. 52, no. 9, pp. 2205-2213, Sept. 2004.

[3] D. Erricolo and P. L. E. Uslenghi, "Exact radiation and scattering for an elliptic metal cylinder at the interface between isorefractive half-spaces," IEEE Trans. Antennas Propagat., vol. 52, no. 9, pp. 2214-2225, Sept. 2004.

[4] D. Erricolo and P. L. E. Uslenghi, "Penetration, radiation, and scattering for a cavity-backed gap in a corner," IEEE Trans. Antennas Propagat., vol. 53, no. 8, pp. 2738-2748, Aug. 2005.

[5] D. Erricolo, M.D. Lockard, C.M. Butler, and P.L.E. Uslenghi, "Comparison among currents on surfaces inside and near a semielliptical channel filled with isorefractive material that backs a slotted plane: currents computed by analytical formulas and by integral equation methods," in Proc. Intl. Conf. on Electromagnetics in Advanced Applications (ICEAA'03), Torino, Italy, Sept 2003, pp. 469-474.

[6] D. Erricolo, M. D. Lockard, C. M. Butler, and P. L. E. Uslenghi, "Numerical analysis of penetration, radiation, and scattering for a $2 \mathrm{D}$ slotted semielliptical channel filled with isorefractive material," PIER, vol. 53, pp. 69-89, 2005.

[7] D. Erricolo, M.D. Lockard, C.M. Butler, and P.L.E. Uslenghi, "Currents on conducting surfaces of a semielliptical-channel-backed slotted screen in an isorefractive environment," IEEE Trans. Antennas Propagat., vol. 53 , no. 7, pp. 2350-2356, July 2005.

[8] J. J. Bowman, T. B. A. Senior, and P. L. E. Uslenghi, Electromagnetic and Acoustic Scattering by Simple Shapes, Hemisphere Publishing Corporation, New York, 1987.

[9] C. Flammer, Spheroidal wave functions, Stanford University Press, 1957.

[10] S. Zhang and J.-M. Jin, Computation of Special Functions, Wiley, New York, 1996.

[11] D. Erricolo, "Acceleration of the convergence of series containing Mathieu functions using Shanks transformation," IEEE Antennas and Wireless Propagation Letters, vol. 2, pp. 58-61, 2003.

[12] C. C. Lin and K. K. Mei, "Radiation and scattering from partially buried vertical wires," Electromagnetics, vol. 2, pp. 309-334, 1982.

[13] G. J. Burke, W. A. Johnson, and E. K. Miller, "Modeling of simple antennas near to and penetrating an interface," Proc. IEEE, vol. 71, no. 1, pp. 74-75, Jan. 1983.

[14] G. J. Burke and E. K. Miller, "Modeling antennas near to and penetrating a lossy interface," IEEE Trans. Antennas Propagat., vol. 32, no. 10, pp. 1040-1049, Oct. 1984

[15] W. A. Johnson, "Analysis of a vertical, tubular cylinder which penetrates an air-dielectric interface and which is excited by an azimuthally symmetric source," Radio Sci., vol. 18, no. 6, pp. 1273-1281, Nov.Dec. 1983. 
[16] C. M. Butler and K. A. Michalski, "Analysis of a cylindrical antenna residing in two contiguous half spaces," in Digest of National Radio Science Meeting, Boulder, CO, USA, 5-7 Jan. 1983, p. 55.

[17] C. M. Butler, W. A. Johnson, and K. A. Michalski, "Radiation and scattering from partially buried objects," in URSI XXI General Assembly, Florence, Italy, Aug.-Sep. 1984.

[18] K. A. Michalski and C. M. Butler, "Evaluation of Sommerfeld integrals arising in the ground stake antenna problem," IEE Proc., Part H, vol. 134, no. 1, pp. 93-97, Feb. 1987.

[19] C. M. Butler, A. Q. Martin, and K. A. Michalski, "Analysis of a cylindrical antenna in a circular aperture in a screen," J. Electromagn. Waves Appl., vol. 8, no. 2, pp. 149-173, 1994. 